International Journal of Pure and Applied Mathematics

Volume 95 No. 1 2014, 79-88

ISSN: 1311-8080 (printed version); ISSN: 1314-3395 (on-line version)

url: http://www.ijpam.eu

doi: http://dx.doi.org/10.12732/ijpam.v95i1.9

ijpam.eu

\title{
EXPONENTIAL STABILITY OF STOCHASTIC HYBRID SYSTEMS WITH NONDIFFERENTIABLE AND INTERVAL TIME-VARYING DELAY
}

\author{
Manlika Rajchakit ${ }^{1}$, Grienggrai Rajchakit ${ }^{2} \S$ \\ ${ }^{1,2}$ Department of Mathematics and Statistics \\ Maejo University \\ Chiangmai, 50290, THAILAND
}

\begin{abstract}
This paper addresses exponential stability problem for a class of stochastic hybrid systems with time-varying delay. The time delay is any continuous function belonging to a given interval, but not necessary to be differentiable. By constructing a suitable augmented Lyapunov-Krasovskii functional combined with Leibniz-Newton's formula, new delay-dependent sufficient conditions for exponential stability of stochastic hybrid systems with time-varying delay are first established in terms of LMIs.
\end{abstract}

AMS Subject Classification: 15A09, 52A10, 74M05, 93D05, 93D20, 94C10 Key Words: switching design, mean square exponential stability, switched stochastic systems, scalar Wiener process, Brownian Motion, interval delay, Lyapunov function, linear matrix inequalities

\section{Introduction}

As an important class of hybrid systems, switched systems arise in many practical processes that cannot be described by exclusively continuous or exclusively discrete models, such as manufacturing, communication networks, automotive

Received: February 17, 2014

(C) 2014 Academic Publications, Ltd.

${ }^{\S}$ Correspondence author url: www.acadpubl.eu 
engineering control and chemical processes (see, e.g., [1-9] and the references therein). The analysis of stochastic systems with respect to mean square stability of their equilibria has attracted many researchers. Such systems occur in a large number of applications as in Physics, Optics or Mechanical Engineering. Often, these systems can generally be written as systems of stochastic differential equations(SDEs). There stability examinations play an essential role in judgement on qualitative behaviour of natural processes. The concept of mean square stability is one of the most attractive and feasible ones within the large branch of stability analysis. To the best of our knowledge, exponential stability of switched stochastic systems with interval time-varying delay, non-differentiable time-varying delays have not been fully studied yet (see, e.g., [10-18] and the references therein). Which are important in both theories and applications. This motivates our research.

This paper gives the improved results for the mean square exponential stability of stochastic hybrid systems with interval time-varying delay. The time delay is assumed to be a time-varying continuous function belonging to a given interval, but not necessary to be differentiable. Specifically, our goal is to develop exponential stability of stochastic hybrid systems with interval timevarying delay. By constructing augmented Lyapunov functional combined with LMI technique, we propose new criteria for the exponential stability of stochastic hybrid systems with interval time-varying delay. The delay-dependent exponential stability conditions are formulated in terms of LMIs.

The paper is organized as follows: Section 2 presents definitions and some well-known technical propositions needed for the proof of the main results. Delay-dependent exponential stability conditions of stochastic hybrid systems with interval time-varying delay is presented in Section 3. The conclusions are drawn in Section 4.

\section{Preliminaries}

The following notations will be used in this paper. $R^{+}$denotes the set of all real non-negative numbers; $R^{n}$ denotes the $n$-dimensional space with the scalar product $\langle.,$.$\rangle and the vector norm \|.\| ; M^{n \times r}$ denotes the space of all matrices of $(n \times r)$-dimensions; $A^{T}$ denotes the transpose of matrix $A ; A$ is symmetric if $A=A^{T} ; I$ denotes the identity matrix; $\lambda(A)$ denotes the set of all eigenvalues of $A ; \lambda_{\min / \max }(A)=\min / \max \{\operatorname{Re} \lambda ; \lambda \in \lambda(A)\} ; x_{t}:=\{x(t+s)$ : $s \in[-h, 0]\},\left\|x_{t}\right\|=\sup _{s \in[-h, 0]}\|x(t+s)\| ; C\left([0, t], R^{n}\right)$ denotes the set of all $R^{n}$-valued continuous functions on $[0, t]$; Matrix $A$ is called semi-positive 
definite $(A \geq 0)$ if $\langle A x, x\rangle \geq 0$, for all $x \in R^{n} ; A$ is positive definite $(A>0)$ if $\langle A x, x\rangle>0$ for all $x \neq 0 ; A>B$ means $A-B>0$. * denotes the symmetric term in a matrix.

Consider a switched stochastic system with interval time-varying delay of the form

$$
\begin{aligned}
& \dot{x}(t)=A_{\gamma(x(t))} x(t)+D_{\gamma(x(t))} x(t-h(t))+\sigma(x(t), x(k-h(t)), t) \omega(t), \\
& t \in R^{+}, x(t)=\phi(t), t \in\left[-h_{2}, 0\right],
\end{aligned}
$$

where $x(t) \in R^{n}$ is the state; $\gamma():. R^{n} \rightarrow \mathcal{N}:=\{1,2, \ldots, N\}$ is the switching rule, which is a function depending on the state at each time and will be designed. A switching function is a rule which determines a switching sequence for a given switching system. Moreover, $\gamma(x(t))=i$ implies that the system realization is chosen as the $i^{\text {th }}$ system, $i=1,2, \ldots, N$. It is seen that the system (1) can be viewed as an autonomous switched system in which the effective subsystem changes when the state $x(t)$ hits predefined boundaries. $A_{i}, D_{i} \in$ $M^{n \times n}, i=1,2, \ldots, N$ are given constant matrices, and $\phi(t) \in C\left(\left[-h_{2}, 0\right], R^{n}\right)$ is the initial function with the norm $\|\phi\|=\sup _{s \in\left[-h_{2}, 0\right]}\|\phi(s)\|$. $\omega(k)$ is a scalar Wiener process (Brownian Motion) on $(\Omega, \mathcal{F}, \mathcal{P})$ with

$$
E\{\omega(t)\}=0, \quad E\left\{\omega^{2}(t)\right\}=1, \quad E\{\omega(i) \omega(j)\}=0(i \neq j),
$$

and $\sigma_{i}: R^{n} \times R^{n} \times R \rightarrow R^{n}, i=1,2, \ldots, N$ is the continuous function, and is assumed to satisfy that

$$
\begin{gathered}
\sigma^{T}(x(t), x(t-h(t)), t) \sigma(x(t), x(t-h(t)), t) \leq \rho_{1} x^{T}(t) x(t) \\
+\rho_{2} x^{T}(t-h(t)) x(t-h(t)), x(t), x(t-h(t)) \in R^{n},
\end{gathered}
$$

where $\rho_{1}>0$ and $\rho_{2}>0$, are known constant scalars. For simplicity, we denote $\sigma(x(t), x(t-h(t)), t)$ by $\sigma$, respectively.

The time-varying delay function $h(t)$ satisfies

$$
0 \leq h_{1} \leq h(t) \leq h_{2}, \quad t \in R^{+} .
$$

The mean square stability problem for switched stochastic system (1) is to construct a switching rule that makes the system mean square exponentially stable.

Definition 1. Given $\alpha>0$. The switched stochastic system (1) is $\alpha$ exponentially stable in the mean square if there exists a switching rule $\gamma($. such that every solution $x(t, \phi)$ of the system satisfies the following condition

$$
\exists N>0: \quad E\{\|x(t, \phi)\|\} \leq E\left\{N e^{-\alpha t}\|\phi\|\right\}, \quad \forall t \in R^{+} .
$$


We end this section with the following technical well-known propositions, which will be used in the proof of the main results.

Definition 2. The system of matrices $\left\{J_{i}\right\}, i=1,2, \ldots, N$, is said to be strictly complete if for every $x \in R^{n} \backslash\{0\}$ there is $i \in\{1,2, \ldots, N\}$ such that $x^{T} J_{i} x<0$.

It is easy to see that the system $\left\{J_{i}\right\}$ is strictly complete if and only if

$$
\bigcup_{i=1}^{N} \alpha_{i}=R^{n} \backslash\{0\}
$$

where

$$
\alpha_{i}=\left\{x \in R^{n}: \quad x^{T} J_{i} x<0\right\}, i=1,2, \ldots, N .
$$

We end this section with the following technical well-known propositions, which will be used in the proof of the main results.

Proposition 1. (see [19]) The system $\left\{J_{i}\right\}, i=1,2, \ldots, N$, is strictly complete if there exist $\delta_{i} \geq 0, i=1,2, \ldots, N, \sum_{i=1}^{N} \delta_{i}>0$ such that

$$
\sum_{i=1}^{N} \delta_{i} J_{i}<0
$$

If $N=2$ then the above condition is also necessary for the strict completeness.

Proposition 2. (Cauchy Inequality) For any symmetric positive definite $\operatorname{marix} N \in M^{n \times n}$ and $a, b \in R^{n}$ we have

$$
\pm a^{T} b \leq a^{T} N a+b^{T} N^{-1} b .
$$

Proposition 3. (see [19]) For any symmetric positive definite matrix $M \in M^{n \times n}$, scalar $\mu>0$ and vector function $\omega:[0, \mu] \rightarrow R^{n}$ such that the integrations concerned are well defined, the following inequality holds

$$
\left(\int_{0}^{\mu} \omega(s) d s\right)^{T} M\left(\int_{0}^{\mu} \omega(s) d s\right) \leq \mu\left(\int_{0}^{\mu} \omega^{T}(s) M \omega(s) d s\right) .
$$


Proposition 4. (see [19]) Let $E, H$ and $F$ be any constant matrices of appropriate dimensions and $F^{T} F \leq I$. For any $\epsilon>0$, we have

$$
E F H+H^{T} F^{T} E^{T} \leq \epsilon E E^{T}+\epsilon^{-1} H^{T} H .
$$

Proposition 5. (Schur Complement Lemma, see [19]) Given constant matrices $X, Y, Z$ with appropriate dimensions satisfying $X=X^{T}, Y=Y^{T}>0$. Then $X+Z^{T} Y^{-1} Z<0$ if and only if

$$
\left(\begin{array}{cc}
X & Z^{T} \\
Z & -Y
\end{array}\right)<0 \quad \text { or } \quad\left(\begin{array}{cc}
-Y & Z \\
Z^{T} & X
\end{array}\right)<0 .
$$

\section{Main Results}

Let us set

$\mathcal{M}_{i}=\left[\begin{array}{ccccc}M_{11} & 0 & 0 & M_{14} & M_{15} \\ * & M_{22} & 0 & M_{24} & 0 \\ * & * & M_{33} & M_{34} & 0 \\ * & * & * & M_{44} & M_{45} \\ * & * & * & * & M_{55}\end{array}\right]$,
$M_{11}=A_{i}^{T} P+P A_{i}+2 \alpha P+2 \rho_{1} I, M_{14}=P D_{i}-S_{1} D_{i}, M_{15}=S_{1}-S_{2} A_{i}$,
$M_{22}=-e^{-2 \alpha h_{2}} U, M_{24}=e^{-2 \alpha h_{2}} U, M_{33}=-e^{-2 \alpha h_{2}} U, M_{34}=e^{-2 \alpha h_{2}} U$,
$M_{44}=-2 e^{-2 \alpha h_{2}} U+2 \rho_{2} I, M_{45}=-S_{2} D_{i}, M_{55}=S_{2}+S_{2}^{T}+\left(h_{2}-h_{1}\right)^{2} U$,
$J_{i}=Q-S_{1} A_{i}-A_{i}^{T} S_{1}^{T}, \alpha_{i}=\left\{x \in R^{n}: x^{T} J_{i} x<0\right\}, i=1,2, \ldots, N$,
$\bar{\alpha}_{1}=\alpha_{1}, \bar{\alpha}_{i}=\alpha_{i} \backslash \bigcup_{j=1}^{i-1} \bar{\alpha}_{j}, i=2,3, \ldots, N, \lambda_{1}=\lambda_{\min }(P), \lambda_{2}=\left(h_{2}-h_{1}\right)^{2} \lambda_{\max }(U)$.

The main result of this paper is summarized in the following theorem.

Theorem 1. Given $\alpha>0$. The zero solution of the switched stochastic system (1) is $\alpha$-exponentially stable in the mean square if there exist symmetric positive definite matrices $U$, and matrices $S_{i}, i=1,2$, such that satisfying the following conditions: 
(i) $\exists \delta_{i} \geq 0, i=1,2, \ldots, N, \quad \sum_{i=1}^{N} \delta_{i}>0: \sum_{i=1}^{N} \delta_{i} J_{i}<0$.

(ii) $\mathcal{M}_{i}<0, \quad i=1,2, \ldots, N$.

The switching rule is chosen as $\gamma(x(t))=i$, whenever $x(t) \in \bar{\alpha}_{i}$. Moreover, the solution $x(t, \phi)$ of the switched stochastic system satisfies

$$
E\{\|x(t, \phi)\|\} \leq E\left\{\sqrt{\frac{\lambda_{2}}{\lambda_{1}}} e^{-\alpha t}\|\phi\|\right\}, \quad \forall t \in R^{+} .
$$

Proof. We consider the following Lyapunov-Krasovskii functional for the system (1)

$$
E\left\{V\left(t, x_{t}\right)\right\}=E\left\{\sum_{i=1}^{2} V_{i}\right\}
$$

where

$$
\begin{aligned}
& V_{1}=x^{T}(t) P x(t) \\
& V_{2}=\left(h_{2}-h_{1}\right) \int_{t-h_{2}}^{t-h_{1}} \int_{t+s}^{t} e^{2 \alpha(\tau-t)} \dot{x}^{T}(\tau) U \dot{x}(\tau) d \tau d s .
\end{aligned}
$$

It easy to check that

$$
E\left\{\lambda_{1}\|x(t)\|^{2}\right\} \leq E\left\{V\left(t, x_{t}\right)\right\} \leq E\left\{\lambda_{2}\left\|x_{t}\right\|^{2}\right\}, \quad \forall t \geq 0
$$

Taking the derivative of $V_{1}$ along the solution of system (1) and taking the mathematical expectation, we obtained

$$
\begin{aligned}
E\left\{\dot{V}_{1}\right\}= & E\left\{2 x^{T}(t) P \dot{x}(t)\right\} \\
= & E\left\{x^{T}(t)\left[A_{i}^{T} P+A_{i} P\right] x(t)+2 x^{T}(t) P D_{i} x(t-h(t))+2 x^{T}(t) P \sigma \omega(t)\right\} \\
E\left\{\dot{V}_{2}\right\} \leq & E\left\{\left(h_{2}-h_{1}\right)^{2} \dot{x}^{T}(t) U \dot{x}(t)\right. \\
& \left.-\left(h_{2}-h_{1}\right) e^{-2 \alpha h_{2}} \int_{t-h_{2}}^{t-h_{1}} \dot{x}^{T}(s) U \dot{x}(s) d s-2 \alpha V_{2}\right\} .
\end{aligned}
$$

Using Proposition 2 gives

$$
\begin{aligned}
& E\left\{\left[h_{2}-h(t)\right] \int_{t-h_{2}}^{t-h(t)} \dot{x}^{T}(s) U \dot{x}(s) d s\right\} \\
& \geq E\left\{\left[\int_{t-h_{2}}^{t-h(t)} \dot{x}(s) d s\right]^{T} U\left[\int_{t-h_{2}}^{t-h(t)} \dot{x}(s) d s\right]\right\} \\
& \geq E\left\{\left[x(t-h(t))-x\left(t-h_{2}\right)\right]^{T} U\left[x(t-h(t))-x\left(t-h_{2}\right)\right]\right\} .
\end{aligned}
$$


Similarly, we have

$$
\begin{aligned}
& E\left\{-\left(h_{2}-h_{1}\right) \int_{t-h(t)}^{t-h_{1}} \dot{x}^{T}(s) U \dot{x}(s) d s\right\} \\
& \leq E\left\{-\left[x\left(t-h_{1}\right)-x(t-h(t))\right]^{T} U\left[x\left(t-h_{1}\right)-x(t-h(t))\right]\right\} .
\end{aligned}
$$

Therefore, we have

$$
\begin{aligned}
& E\{\dot{V}(.)+2 \alpha V(.)\} \\
& \leq E\left\{x^{T}(t)\left[A_{i}^{T} P+P A_{i}+2 \alpha P\right\}+E\left\{x^{T}(t)\left[S_{1} A_{i}+A_{i}^{T} S_{1}^{T}+2 \rho_{1} I\right] x(t)\right\}\right. \\
& +E\left\{2 x^{T}(t)\left[P D_{i}-S_{1} D_{i} x(t-h(t))\right\}+E\left\{2 x^{T}(t)\left[S_{1}-S_{2} A_{i}\right] \dot{x}(t)\right\}\right. \\
& +E\left\{x^{T}\left(t-h_{1}\right)\left[-e^{-2 \alpha h_{2}} U\right] x\left(t-h_{1}\right)\right\}+E\left\{2 x^{T}\left(t-h_{1}\right)\left[e^{-2 \alpha h_{2}} U\right] x(t-h(t))\right\} \\
& +E\left\{x^{T}\left(t-h_{2}\right)\left[-e^{-2 \alpha h_{2}} U\right] x\left(t-h_{2}\right)\right\}+E\left\{x^{T}\left(t-h_{2}\right)\left[e^{-2 \alpha h_{2}} U\right] x(t-h(t))\right\} \\
& +E\left\{x^{T}(t-h(t))\left[-2 e^{-2 \alpha h_{2}} U+2 \rho_{2} I\right] x(t-h(t))\right\} \\
& +E\left\{2 x^{T}(t-h(t))\left[-S_{2} D_{i}\right] \dot{x}(t)\right\} \\
& +E\left\{\dot{x}^{T}(t)\left[S_{2}+S_{2}^{T}+\left(h_{2}-h_{1}\right)^{2} U\right] \dot{x}(t)\right\} \\
& =E\left\{x^{T}(t) J_{i} x(t)+\zeta^{T}(t) \mathcal{M}_{i} \zeta(t)\right\}
\end{aligned}
$$

where

$$
\zeta(t)=\left[x(t), x\left(t-h_{1}\right), x\left(t-h_{2}\right), x(t-h(t)), \dot{x}(t)\right] .
$$

Therefore, we finally obtain from (6) and the condition (ii) that

$$
E\{\dot{V}(.)+2 \alpha V(.)\}<E\left\{x^{T}(t) J_{i} x(t)\right\}, \quad \forall i=1,2, \ldots ., N, \quad t \in R^{+} .
$$

We now apply the condition (i) and Proposition 1, the system $J_{i}$ is strictly complete, and the sets $\alpha_{i}$ and $\bar{\alpha}_{i}$ by (4) are well defined such that

$$
\begin{gathered}
\bigcup_{i=1}^{N} \alpha_{i}=R^{n} \backslash\{0\}, \\
\bigcup_{i=1}^{N} \bar{\alpha}_{i}=R^{n} \backslash\{0\}, \quad \bar{\alpha}_{i} \cap \bar{\alpha}_{j}=\emptyset, i \neq j .
\end{gathered}
$$


Therefore, for any $x(t) \in R^{n}, \quad t \in R^{+}$, there exists $i \in\{1,2, \ldots, N\}$ such that $x(t) \in \bar{\alpha}_{i}$. By choosing switching rule as $\gamma(x(t))=i$ whenever $\gamma(x(t)) \in \bar{\alpha}_{i}$, from (6) we have

$$
E\{\dot{V}(.)+2 \alpha V(.)\} \leq E\left\{x^{T}(t) J_{i} x(t)\right\}<0, \quad t \in R^{+},
$$

and hence

$$
E\left\{\dot{V}\left(t, x_{t}\right)\right\} \leq E\left\{-2 \alpha V\left(t, x_{t}\right)\right\}, \quad \forall t \in R^{+} .
$$

Integrating both sides of (7) from 0 to $t$, we obtain

$$
E\left\{V\left(t, x_{t}\right)\right\} \leq E\left\{V(\phi) e^{-2 \alpha t}\right\}, \quad \forall t \in R^{+} .
$$

Furthermore, taking condition (5) into account, we have

$$
E\left\{\lambda_{1}\|x(t, \phi)\|^{2}\right\} \leq E\left\{V\left(x_{t}\right)\right\} \leq E\left\{V(\phi) e^{-2 \alpha t}\right\} \leq E\left\{\lambda_{2} e^{-2 \alpha t}\|\phi\|^{2}\right\},
$$

then

$$
E\{\|x(t, \phi)\|\} \leq E\left\{\sqrt{\frac{\lambda_{2}}{\lambda_{1}}} e^{-\alpha t}\|\phi\|\right\}, \quad t \in R^{+},
$$

By Definition 1, the system (1) is exponentially stable in the mean square. The proof is complete.

\section{Conclusion}

In this paper, we have proposed new delay-dependent conditions for exponential stability of stochastic hybrid systems with time-varying delay. Based on the improved Lyapunov-Krasovskii functional and linear matrix inequality technique, a switching rule for exponential stability of stochastic hybrid systems with timevarying delay have been established in terms of LMIs.

\section{Acknowledgements}

This work was supported by the Thailand Research Fund Grant, the Commission for Higher Education and Faculty of Science, Maejo University, Thailand. 


\section{References}

[1] Y.J. Sun, Global stabilizability of uncertain systems with time-varying delays via dynamic observer-based output feedback, Linear Algebra and its Applications, 353(2002), 91-105.

[2] Kreangkri Ratchagit, STABILITY CRITERIA OF LPD SYSTEM WITH TIME-VARYING DELAY, International Journal of Pure and Applied Mathematics, Vol. 78 No. 6, 2012, 857-866.

[3] G. Rajchakit, Stabilization of switched discrete-time systems with convex polytopic uncertainties, Journal of Computational Analysis \& Applications 16(2014), 20-29.

[4] VN. Phat, Y. Kongtham, and K. Ratchagit, LMI approach to exponential stability of linear systems with interval time-varying delays, Linear Algebra Appl., Vol. 436, pp. 243-251, 2012. doi: 10.1016/j.laa.2011.07.016

[5] K. Ratchagit, V.N. Phat, Stability and stabilization of switched linear discrete-time systems with interval time-varying delay, Nonlinear Anal. Hybrid Syst. 5 (2011) 605-612. DOI: 10.1016/j.nahs.2011.05.006

[6] K. Ratchagit and V.N. Phat, Stability criterion for discrete-time systems, J. Ineq. Appl., 2010(2010), 1-6. doi:10.1155/2010/201459

[7] Zidong Wang, Yao Wang, and Yurong Liu, Global Synchronization for Discrete-Time Stochastic Complex Networks With Randomly Occurred Nonlinearities and Mixed Time Delays, IEEE Transactions on Neural Networks, 21(2010), 11-25.

[8] M. De la Sen and A. Ibeas, Stability Results of a Class of Hybrid Systems under Switched Continuous-Time and Discrete-Time Control, Discrete Dynamics in Nature and Society, 2009(2009).

[9] K. Ratchagit, Asymptotic stability of nonlinear delay-difference system via matrix inequalities and application, International Journal of Computational Methods, pp. 389-397, 2009. DOI: 10.1142/S0219876209001899

[10] K. Ratchagit and V. N. Phat, Robust Stability and Stabilization of Linear Polytopic Delay-Difference Equations with Interval Time-Varying Delays, Neural, Parallel, and Scientific Computations, 19(2011), 361-372. 
[11] Kreangkri Ratchagit , STABILITY ANALYSIS OF LINEAR SYSTEMS WITH TIME DELAYS, International Journal of Pure and Applied Mathematics, Vol. 76 No. 1, 2012, 21-28.

[12] K. Ratchagit , THE SUFFICIENT CONDITIONS FOR STABILITY OF LINEAR TIME-VARYING SYSTEMS WITH STATE DELAYS, International Journal of Pure and Applied Mathematics, Vol. 65 No. 1, 2010, $65-72$.

[13] K. Ratchagit and V.N. Phat, Stability criterion for discrete-time systems, J. Ineq. Appl., 2010(2010), 1-6.

[14] Grienggrai Rajchakit, Delay-Dependent Asymptotical Stabilization Criterion of Recurrent Neural Networks, Applied Mechanics and Materials. 330(2013) 1045-1048. doi:10.4028/www.scientific.net/AMM.330.1045

[15] D. H. Ji, J. H. Park, W. J. Yoo and S. C. Won, Robust memory state feedback model predictive control for discrete-time uncertain state delayed systems, Appl. Math. Comp. 215 (2009) 2035-2044.

[16] Kreangkri Ratchagit , EXPONENTIAL STABILITY OF SWITCHED LINEAR SYSTEMS, International Journal of Pure and Applied Mathematics, Vol. 58 No. 3, 2010, 361-371.

[17] P. Niamsup, G. Rajchakit, New Results on Robust Stability and Stabilization of Linear Discrete-Time Stochastic Systems with Convex Polytopic Uncertainties, JOURNAL OF APPLIED MATHEMATICS, 2013(2013). DOI: $10.1155 / 2013 / 368259$

[18] G. Rajchakit, Stabilization of switched discrete-time systems with convex polytopic uncertainties, Journal of Computational Analysis and Applications 16 (2014) 20-29.

[19] R. P. Agarwal, Difference Equations and Inequalities, Second Edition, Marcel Dekker, New York, 2000. 